\title{
Kanat Gövde Tipindeki Bir İnsansız Hava Aracının Yapısal Tasarımı ve Analizi
}

\author{
Aybuke Gökçen Yılmaz İlaslan ${ }^{1 *}$, İlyas Kandemir ${ }^{1}$ \\ 1*Makine Mühendisliği, Gebze Teknik Üniversitesi, Türkiye, ORCID ID 0000-0001-9995-9163, aybukegokcenyilmaz@gmail.com \\ ${ }^{1}$ Makine Mühendisliği, Gebze Teknik Üniversitesi, Türkiye, ORCID ID 0000-0002-8773-6541, kandemir@gtu.edu.tr
}

(1st International Conference on Applied Engineering and Natural Sciences ICAENS 2021, November 1-3, 2021)

(DOI: 10.31590/ejosat.1011249)

ATIF/REFERENCE: Yılmaz İlaslan, A.G. \& Kandemir, İ. (2021). Kanat Gövde Tipindeki Bir İnsansız Hava Aracının Yapısal Tasarımı ve Analizi. European Journal of Science and Technology, (28), 775-780.

$\ddot{\mathbf{O} z}$

Havacılık alanında İnsansız Hava Araçlarına (IHHA) yönelik talep ve çalışmalar tüm dünyada ve ülkemizde gün geçtikçe artmaktadır. Kullanım amacına uygun olarak, bu araçların verimliliği giderek artmaktadır. Gelişmelerin daha da ilerlemesi için birçok ülke hala insansız hava aracı üzerine birçok araştırma ve çalışma yapmayı sürdürmektedir. Bu çalışmada ilk önce gerekli literatür araştırmaları yapılmış ve bu doğrultuda modellenecek olan kanat gövde tipindeki İHA'nın boyutu ve özellikleri belirlenmiştir. Polistiren Köpük malzemeden yapılan kanat gövde tipindeki bir İHA statik analizleri yapılmak üzere sonlu elemanlar metodu ile çalı̧an ANSYS Workbench programına aktarılmıştır. İHA modelimiz Epoxy Carbon UD, Epoxy E Glass UD ve Epoxy S Glass UD olmak üzere 3 farklı kompozit malzeme ile kaplanmıştır. Kaplanan malzeme kalınlıkları $0,23 \mathrm{~mm}$ ile $0,30 \mathrm{~mm}$ 'dir ve $50 \mathrm{G}$ ile $100 \mathrm{G}$ gibi yüksek $\mathrm{G}$ değerleri uygulanarak analizler gerçekleştirilmiştir. Analizlerde; model üzerindeki gerilmeler, güvenlik faktörü ve kaplanan kompozit malzemelerin üzerinde oluşan gerilmeler ile hasar kriteri incelenmiştir. Elde edilen verilerde kaplanan kompozit malzemerin yüksek G değerlerinde yapılan analizlerinde mukavemetinin tek katlı kaplamada bile yeterince yüksek olduğu gösterilmiştir.

Anahtar Kelimeler: İha, Kompozit Malzeme, Statik Analiz

\section{Structural Design and Analysis of A Wing-Body Type Unmanned Aircraft}

\begin{abstract}
Demand and studies for Unmanned Aerial Vehicles (UAV) in the field of aviation are increasing day by day all over the world and in our country. In accordance with the intended use, the efficiency of these tools is gradually increasing. In order to further progress, many countries still continue to conduct many researches and studies on unmanned aerial vehicles. In this study, firstly, the necessary literature research was carried out and the size and characteristics of the wing body type UAV to be modeled in this direction were determined. A wing body type UAV made of Polysyrene Foam material was transferred to the ANSYS Workbench program, which works with the finite element method, for static analysis. Our UAV model is covered with 3 different composite materials: Epoxy Carbon UD, Epoxy E Glass UD and Epoxy S Glass UD. The thickness of the coated material is between 0,23 $\mathrm{mm}$ and $0,30 \mathrm{~mm}$, and analyzes were carried out by applying high $\mathrm{G}$ values such as $50 \mathrm{G}$ and $100 \mathrm{G}$. In analysis; The stresses on the model, the safety factor and the stresses on the coated composite materials and the damage criterion were examined. In the analysis of the coated composite material at high $\mathrm{G}$ values in the obtained data, it has been shown that the strength is high enough even in a single layer coating.
\end{abstract}

Keywords: Uav, Composite Material, Statıc Analysis 


\section{Giriş}

IHHA gövde ve kanatlarında kullanılan malzemeler, kanat yapıları, kompozit malzemeler ve üretim yöntemleri ile ilgili yapılan literatür çalışmaları incelenmiştir.

Literatürlere bakıldığında iha gövde kanat profillerinde verim sağlayan kompozit malzemeler kullanılmaktadır. Birçok benzer çalışmalarda ise metal kullanımı olduğu incelenmiştir.

İha'ların düşük hızlarda dahi verimli çalışabilmesi için kanat geometrisi, malzemesi ve kanadın mukavemeti önemlidir.

Maliyet açısından daha ucuz ve geliştirilebilir olması nedeniyle İHA'lar askeri operasyonlar dışında günümüzde son zamanlarda sivil amaçlar için kullanılmaktadır.

Bu çalışmada, NACA 2308 kanat gövde profilinde, köpük malzemeden yapılmış olan modelimiz Epoxy Carbon UD, Epoxy E Glass UD ve Epoxy S Glass UD olmak üzere 3 farklı kompozit malzeme ile kaplanmıştır. $50 \mathrm{G}$ ve $100 \mathrm{G}$ gibi yüksek $\mathrm{G}$ değerleri

\section{Araştırma Sonuçları ve Tartışma}

Polistiren Köpük malzemesinden yapılan model, sabit hızda seyir halindeyken $50 \mathrm{G}$ ve $100 \mathrm{G}$ 'lik sert çarpmaya maruz kaldığında üzerinden oluşan deformasyon ve güvenlik faktörü değerine bakılmıştır. Ardından 3 farklı kompozit malzeme ile kaplanmış ve analiz sonuçları incelenmiştir. Deformasyon değeri ve hasar kriteri değeri polistiren köpük malzeme üzerinde yapılan analizde çok yüksek değerde iken, kompozit kaplama yapıldıktan sonra önemli ölçüde düşüş meydana geldiği gözlenmiştir. Kompozit malzeme olarak seçilen Epoxy S Glass UD, Epoxy E Glass UD ve Epoxy Carbon UD malzemeleri arasında $100 \mathrm{G}$ ve $50 \mathrm{G}$ değerlerinde en yüksek deformasyon değerine sahip olan malzeme Epoxy S Glass UD olarak görülmüştür. $50 \mathrm{G}$ için tek kat kaplama sonuçları aşağıda gösterilmiştir.

Tablo $1.23 \mathrm{~mm} 1$ kat Epoksi Karbon Kaplama

\begin{tabular}{|c|c|c|c|c|}
\hline Kalınlik & & $0,23 \mathrm{~mm}$ & $0,23 \mathrm{~mm}$ & $0,23 \mathrm{~mm}$ \\
\hline \multirow[t]{2}{*}{ Ağ̈rlık } & $0,150 \mathrm{~kg}$ & \multicolumn{3}{|c|}{$0,141 \mathrm{~kg}+0,150 \mathrm{~kg}=0,291 \mathrm{~kg} 0,141 \mathrm{~kg}+0,150 \mathrm{~kg}=0,291 \mathrm{~kg} 0,141 \mathrm{~kg}+0,150 \mathrm{~kg}=0,291 \mathrm{~kg}$} \\
\hline & \begin{tabular}{|l} 
Polistiren Köpük \\
Malzemesi
\end{tabular} & $\begin{array}{l}1 \text { Kat Epoksi Karbon Kaplama } \\
/ 90 \text { derece fiber yönlü }\end{array}$ & $\begin{array}{l}\text { Kaplama/45 derece fiber } \\
\text { yönlü }\end{array}$ & \begin{tabular}{|l} 
Kaplama /0 derece fiber \\
yönlü
\end{tabular} \\
\hline Total Deformasyon & $72,39 \mathrm{~mm}$ & $1,22 \mathrm{~mm}$ & $2,75 \mathrm{~mm}$ & $4,38 \mathrm{~mm}$ \\
\hline XYönündeki deformasyc & $0,36 \mathrm{~mm}$ & $0,040 \mathrm{~mm}$ & $0,089 \mathrm{~mm}$ & $0,069 \mathrm{~mm}$ \\
\hline Y Yönündeki deformasyc & $72,38 \mathrm{~mm}$ & $1,22 \mathrm{~mm}$ & $2,748 \mathrm{~mm}$ & $4,38 \mathrm{~mm}$ \\
\hline 2 Yönündeki deformasyc & $2,73 \mathrm{~mm}$ & $0,010 \mathrm{~mm}$ & $0,0957 \mathrm{~mm}$ & $0,165 \mathrm{~mm}$ \\
\hline Güvenlik Faktörü & 0,778 & 10,73 & 9,63 & 9,33 \\
\hline Kalınlik & & $0,30 \mathrm{~mm}$ & $0,30 \mathrm{~mm}$ & $0,30 \mathrm{~mm}$ \\
\hline \multirow[t]{2}{*}{ Ağrllık } & $0,150 \mathrm{~kg}$ & \multicolumn{3}{|c|}{$0,184 \mathrm{~kg}+0,150 \mathrm{~kg}=0,334 \mathrm{~kg} 0,184 \mathrm{~kg}+0,150 \mathrm{~kg}=0,334 \mathrm{~kg} 0,184 \mathrm{~kg}+0,150 \mathrm{~kg}=0,334 \mathrm{~kg}$} \\
\hline & $\begin{array}{l}\text { Polistiren Köpük } \\
\text { Malzemesi }\end{array}$ & $\begin{array}{l}1 \text { Kat Epoksi Karbon Kaplama } \\
/ 90 \text { derece fiber yönlü }\end{array}$ & $\begin{array}{l}\text { Kaplama /45 derece fiber } \\
\text { yönlü }\end{array}$ & $\begin{array}{l}\text { Kaplama /0 derece fiber } \\
\text { yönlü }\end{array}$ \\
\hline Total Deformasyon & $72,39 \mathrm{~mm}$ & $1,218 \mathrm{~mm}$ & $2,539 \mathrm{~mm}$ & $4,024 \mathrm{~mm}$ \\
\hline XYönündeki deformasy & $0,36 \mathrm{~mm}$ & $0,040 \mathrm{~mm}$ & $0,079 \mathrm{~mm}$ & $0,065 \mathrm{~mm}$ \\
\hline YYönündeki deformasyc & $72,38 \mathrm{~mm}$ & $1,217 \mathrm{~mm}$ & $2,537 \mathrm{~mm}$ & $4,024 \mathrm{~mm}$ \\
\hline 2 Yönündeki deformasyo & $2,73 \mathrm{~mm}$ & $0,008 \mathrm{~mm}$ & $0,085 \mathrm{~mm}$ & $0,146 \mathrm{~mm}$ \\
\hline Güvenlik Faktörü & 0,778 & 9,92 & 9,82 & 9,04 \\
\hline
\end{tabular}

uygulanarak analizler gerçekleştirilmiş̧ir. Analizlerden elde edilen verilere bakıldığında, köpük üzerine kaplanan kompozit malzemelerin mukavemetinin tek katlı kaplamada bile yeterince yüksek olduğu gösterilmiştir.

\section{Materyal ve Metot}

Çalışma için öncelikli olarak insansız hava aracımızın kanat gövde tipindeki tasarımı belirlenmiştir. Bu tasarım belirlendikten sonra İHA modeli SolidWorks programı aracılığı ile modellenmiş̧ir. Modellenen kanat gövde tipindeki İHA'nın analiz sonuçlarını elde edebilmek için model ANSYS Workbench programına aktarılmıştır. ANSYS Workbench'te tasarım şartları verilmiş ve ilgili malzeme tanımlamaları yapılarak analiz gerçekleştirilmiştir. Ansys programında kompozit malzeme ile kaplanan model için ACP modüllerinden faydalanılmıştır. Modelin statik analizini gerçekleştirirken sonlu elemanlar yöntemi kullanılmıştır.

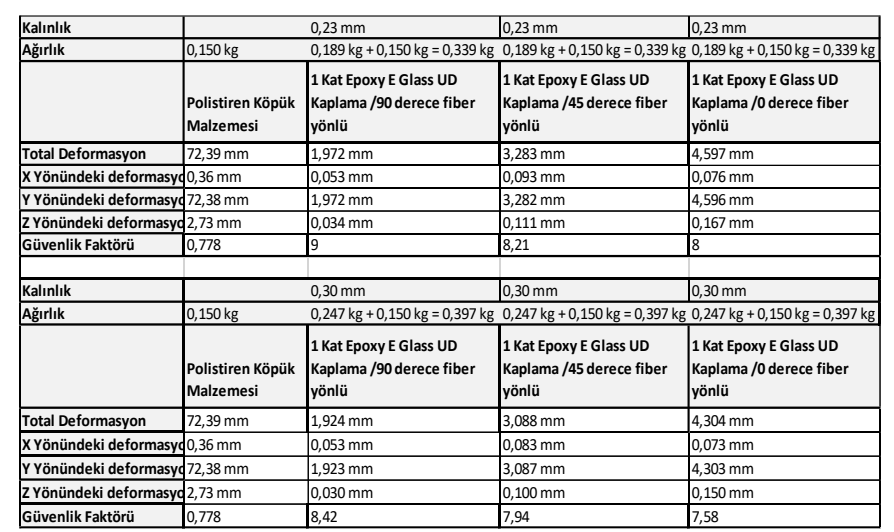

Tablo 3. $23 \mathrm{~mm} 1$ kat Epoksi S Glass UD Kaplama

\begin{tabular}{|c|c|c|c|c|}
\hline Kalınlik & & $0,23 \mathrm{~mm}$ & $0,23 \mathrm{~mm}$ & $0,23 \mathrm{~mm}$ \\
\hline \multirow[t]{2}{*}{ Ağ̈rllık } & $0,150 \mathrm{~kg}$ & $0,189 \mathrm{~kg}+0,150 \mathrm{~kg}=0,337 \mathrm{~kg}$ & $0,189 \mathrm{~kg}+0,150 \mathrm{~kg}=0,339 \mathrm{~kg}$ & $0,189 \mathrm{~kg}+0,150 \mathrm{~kg}=0,339 \mathrm{~kg}$ \\
\hline & $\begin{array}{l}\text { Polistiren Köpük } \\
\text { Malzemesi }\end{array}$ & $\begin{array}{l}1 \text { Kat S Glass Kaplama/90 } \\
\text { derece fiber yönlü }\end{array}$ & $\begin{array}{l}1 \text { Kat S Glass Kaplama /45 } \\
\text { derece fiber yönlü }\end{array}$ & $\begin{array}{l}1 \text { Kat S Glass Kaplama /0 } \\
\text { derece fiber yönlü }\end{array}$ \\
\hline Total Deformasyon & $72,39 \mathrm{~mm}$ & $1,889 \mathrm{~mm}$ & $3,559 \mathrm{~mm}$ & $5,399 \mathrm{~mm}$ \\
\hline XYönündeki deformasycc & $0,36 \mathrm{~mm}$ & $0,052 \mathrm{~mm}$ & $0,111 \mathrm{~mm}$ & $0,082 \mathrm{~mm}$ \\
\hline Y Yönündeki deformasyc & $72,38 \mathrm{~mm}$ & $1,888 \mathrm{~mm}$ & $3,557 \mathrm{~mm}$ & $5,398 \mathrm{~mm}$ \\
\hline 2 Yönündeki deformasyq & $2,73 \mathrm{~mm}$ & $0,030 \mathrm{~mm}$ & $0,117 \mathrm{~mm}$ & $0,208 \mathrm{~mm}$ \\
\hline Güvenlik Faktörü & 0,778 & 9,02 & 8,3 & 7,6 \\
\hline Kalınlık & & $0,30 \mathrm{~mm}$ & $0,30 \mathrm{~mm}$ & $0,30 \mathrm{~mm}$ \\
\hline \multirow[t]{2}{*}{ Ağ̈rllk } & $0,150 \mathrm{~kg}$ & $0,247 \mathrm{~kg}+0,150 \mathrm{~kg}=0,397 \mathrm{~kg}$ & $0,247 \mathrm{~kg}+0,150 \mathrm{~kg}=0,397 \mathrm{~kg}$ & $0,247 \mathrm{~kg}+0,150 \mathrm{~kg}=0,397 \mathrm{~kg}$ \\
\hline & $\begin{array}{l}\text { Polistiren Köpük } \\
\text { Malzemesi }\end{array}$ & $\begin{array}{l}1 \text { Kat S Glass Kaplama/90 } \\
\text { derece fiber yönlü }\end{array}$ & $\begin{array}{l}1 \text { Kat S Glass Kaplama /45 } \\
\text { derece fiber yönlü }\end{array}$ & $\begin{array}{l}1 \text { Kat S Glass Kaplama /0 } \\
\text { derece fiber yönlü }\end{array}$ \\
\hline Total Deformasyon & $72,39 \mathrm{~mm}$ & $1,846 \mathrm{~mm}$ & $3,343 \mathrm{~mm}$ & $5,034 \mathrm{~mm}$ \\
\hline X Yönündeki deformasycc & $0,36 \mathrm{~mm}$ & $0,052 \mathrm{~mm}$ & $0,098 \mathrm{~mm}$ & $0,078 \mathrm{~mm}$ \\
\hline Y Yönündeki deformasyc & $72,38 \mathrm{~mm}$ & $1,845 \mathrm{~mm}$ & $3,341 \mathrm{~mm}$ & $5,034 \mathrm{~mm}$ \\
\hline ZYönündeki deformasyg 2 & $2,73 \mathrm{~mm}$ & $0,027 \mathrm{~mm}$ & $0,106 \mathrm{~mm}$ & $0,189 \mathrm{~mm}$ \\
\hline Güvenlik Faktörü & 0,778 & 8,41 & 7,9 & 7,3 \\
\hline
\end{tabular}


İki kat kaplama sonuçları aşağıda gösterilmiştir.

Tablo 4. $23 \mathrm{~mm} 2$ kat Epoksi Karbon Kaplama

\begin{tabular}{|c|c|c|c|c|}
\hline Kalınlık & & $0,23 \mathrm{~mm}$ & $0,23 \mathrm{~mm}$ & $0,23 \mathrm{~mm}$ \\
\hline Ağ̈rlık & $0,150 \mathrm{~kg}$ & $0,282 \mathrm{~kg}+0,150 \mathrm{~kg}=0,432 \mathrm{~kg}$ & $0,282 \mathrm{~kg}+0,150 \mathrm{~kg}=0,432 \mathrm{~kg}$ & $\mathrm{~g} 0,282 \mathrm{~kg}+0,150 \mathrm{~kg}=0,432 \mathrm{~kg}$ \\
\hline 0 derece atak açı & $\begin{array}{l}\text { Polistiren Köpük } \\
\text { Malzemesi }\end{array}$ & $\begin{array}{l}2 \text { Kat Epoksi Karbon Kaplama } \\
\text { /90 derece fiber yönlü }\end{array}$ & $\begin{array}{l}2 \text { Kat Epoksi Karbon } \\
\text { Kaplama /45 derece fiber } \\
\text { yönlü }\end{array}$ & $\begin{array}{l}\text { 2 Kat Epoksi Karbon } \\
\text { Kaplama /0 derece fiber } \\
\text { yönlü }\end{array}$ \\
\hline Total Deformasyon & $72,39 \mathrm{~mm}$ & $1,23 \mathrm{~mm}$ & $2,31 \mathrm{~mm}$ & $3,62 \mathrm{~mm}$ \\
\hline XYönündeki deformasy & $0,36 \mathrm{~mm}$ & $0,042 \mathrm{~mm}$ & $0,067 \mathrm{~mm}$ & $0,060 \mathrm{~mm}$ \\
\hline Y Yönündeki deformasy & $72,38 \mathrm{~mm}$ & $1,23 \mathrm{~mm}$ & $2,31 \mathrm{~mm}$ & $3,62 \mathrm{~mm}$ \\
\hline Z Yönündeki deformasyc & $2,73 \mathrm{~mm}$ & $0,006 \mathrm{~mm}$ & $0,073 \mathrm{~mm}$ & $0,123 \mathrm{~mm}$ \\
\hline Güvenlik Faktörü & 0,778 & 8,4 & 9,27 & 8,25 \\
\hline Kalınlık & & $0,30 \mathrm{~mm}$ & $0,30 \mathrm{~mm}$ & $0,30 \mathrm{~mm}$ \\
\hline Ağırlık & $0,150 \mathrm{~kg}$ & $0,368 \mathrm{~kg}+0,150 \mathrm{~kg}=0,518 \mathrm{~kg}$ & $0,368 \mathrm{~kg}+0,150 \mathrm{~kg}=0,518 \mathrm{~kg}$ & $\mathrm{~g} 0,368 \mathrm{~kg}+0,150 \mathrm{~kg}=0,518 \mathrm{~kg}$ \\
\hline 0 derece atak açı & $\begin{array}{l}\text { Polistiren Köpük } \\
\text { Malzemesi }\end{array}$ & $\begin{array}{l}\text { 2 Kat Epoksi Karbon Kaplama } \\
\text { /90 derece fiber yönlü }\end{array}$ & $\begin{array}{l}2 \text { Kat Epoksi Karbon } \\
\text { Kaplama /45 derece fiber } \\
\text { yönlü }\end{array}$ & $\begin{array}{l}2 \text { Kat Epoksi Karbon } \\
\text { Kaplama /0 derece fiber } \\
\text { yönlü }\end{array}$ \\
\hline Total Deformasyon & $72,39 \mathrm{~mm}$ & $1,26 \mathrm{~mm}$ & $2,21 \mathrm{~mm}$ & $3,46 \mathrm{~mm}$ \\
\hline XYönündeki deformasy & $0,36 \mathrm{~mm}$ & $0,044 \mathrm{~mm}$ & $0,063 \mathrm{~mm}$ & $0,059 \mathrm{~mm}$ \\
\hline Y Yönündeki deformasy & $72,38 \mathrm{~mm}$ & $1,26 \mathrm{~mm}$ & $2,21 \mathrm{~mm}$ & $3,46 \mathrm{~mm}$ \\
\hline ZYönündeki deformasyc & $2,73 \mathrm{~mm}$ & $0,006 \mathrm{~mm}$ & $0,068 \mathrm{~mm}$ & $0,113 \mathrm{~mm}$ \\
\hline Güvenlik Faktörü & 0,778 & 7,63 & 8,73 & 7,72 \\
\hline
\end{tabular}

Tablo 5. $23 \mathrm{~mm} 2$ kat Epoksi E Glass UD Kaplama

\begin{tabular}{|c|c|c|c|c|}
\hline Kalınlık & & $0,23 \mathrm{~mm}$ & $0,23 \mathrm{~mm}$ & $0,23 \mathrm{~mm}$ \\
\hline Ağırlık & $0,150 \mathrm{~kg}$ & $0,379 \mathrm{~kg}+0,150 \mathrm{~kg}=0,529 \mathrm{~kg}$ & $0,379 \mathrm{~kg}+0,150 \mathrm{~kg}=0,529 \mathrm{~kg}$ & $\mathrm{~g} 0,379 \mathrm{~kg}+0,150 \mathrm{~kg}=0,529 \mathrm{~kg}$ \\
\hline 0 derece atak açı & $\begin{array}{l}\text { Polistiren Köpük } \\
\text { Malzemesi }\end{array}$ & $\begin{array}{l}2 \text { Kat Epoxy E Glass UD } \\
\text { Kaplama/90 derece fiber } \\
\text { yönlü }\end{array}$ & $\begin{array}{l}\text { 2Kat Epoxy E Glass UD } \\
\text { Kaplama /45 derece fiber } \\
\text { yönlü }\end{array}$ & $\begin{array}{l}2 \text { Kat Epoxy E Glass UD } \\
\text { Kaplama /0 derece fiber } \\
\text { yönlü }\end{array}$ \\
\hline Total Deformasyon & $72,39 \mathrm{~mm}$ & $1,90 \mathrm{~mm}$ & $2,88 \mathrm{~mm}$ & $4,00 \mathrm{~mm}$ \\
\hline \begin{tabular}{|l} 
XYönündeki deformasyc \\
\end{tabular} & $0,36 \mathrm{~mm}$ & $0,054 \mathrm{~mm}$ & $0,072 \mathrm{~mm}$ & $0,070 \mathrm{~mm}$ \\
\hline YYönündeki deformasyc & $72,38 \mathrm{~mm}$ & $1,90 \mathrm{~mm}$ & $2,88 \mathrm{~mm}$ & $4,00 \mathrm{~mm}$ \\
\hline ZYönündeki deformasyo & $2,73 \mathrm{~mm}$ & $0,025 \mathrm{~mm}$ & $0,088 \mathrm{~mm}$ & $0,131 \mathrm{~mm}$ \\
\hline Güvenlik Faktörü & 0,778 & 7,05 & 7,35 & 6,95 \\
\hline Kalınlık & & $0,30 \mathrm{~mm}$ & $0,30 \mathrm{~mm}$ & $0,30 \mathrm{~mm}$ \\
\hline Ağrrlık & $0,150 \mathrm{~kg}$ & $0,494 \mathrm{~kg}+0,150 \mathrm{~kg}=0,644 \mathrm{~kg}$ & $0,494 \mathrm{~kg}+0,150 \mathrm{~kg}=0,644 \mathrm{~kg}$ & $\mathrm{~g} 0,494 \mathrm{~kg}+0,150 \mathrm{~kg}=0,644 \mathrm{~kg}$ \\
\hline 0 derece atak açı & $\begin{array}{l}\text { Polistiren Köpük } \\
\text { Malzemesi }\end{array}$ & $\begin{array}{l}2 \text { Kat Epoxy E Glass UD } \\
\text { Kaplama/90 derece fiber } \\
\text { yönlü }\end{array}$ & $\begin{array}{l}\begin{array}{l}2 \text { Kat Epoxy E Glass UD } \\
\text { Kaplama /45 derece fiber } \\
\text { yönlü }\end{array} \\
\end{array}$ & $\begin{array}{l}2 \text { Kat Epoxy E Glass UD } \\
\text { Kaplama/0 derece fiber } \\
\text { yönlü }\end{array}$ \\
\hline Total Deformasyon & $72,39 \mathrm{~mm}$ & $1,92 \mathrm{~mm}$ & $2,81 \mathrm{~mm}$ & $3,88 \mathrm{~mm}$ \\
\hline \begin{tabular}{|l|} 
XYönündeki deformasyc \\
\end{tabular} & $0,36 \mathrm{~mm}$ & $0,056 \mathrm{~mm}$ & $0,068 \mathrm{~mm}$ & $0,069 \mathrm{~mm}$ \\
\hline YYönündeki deformasyc & $772,38 \mathrm{~mm}$ & $1,92 \mathrm{~mm}$ & $2,81 \mathrm{~mm}$ & $3,88 \mathrm{~mm}$ \\
\hline Z Yönündeki deformasyo & $2,73 \mathrm{~mm}$ & $0,022 \mathrm{~mm}$ & $0,083 \mathrm{~mm}$ & $0,122 \mathrm{~mm}$ \\
\hline Güvenlik Faktörü & 0,778 & 6,24 & 6,72 & 6,3 \\
\hline
\end{tabular}

Tablo 6. $23 \mathrm{~mm} 2$ kat Epoksi S Glass UD Kaplama

\begin{tabular}{|c|c|c|c|c|}
\hline Kalınlik & & $0,23 \mathrm{~mm}$ & $0,23 \mathrm{~mm}$ & $0,23 \mathrm{~mm}$ \\
\hline Ağrrlık & $0,150 \mathrm{~kg}$ & $0,379 \mathrm{~kg}+0,150 \mathrm{~kg}=0,529 \mathrm{~kg}$ & $0,379 \mathrm{~kg}+0,150 \mathrm{~kg}=0,529 \mathrm{~kg}$ & $0,379 \mathrm{~kg}+0,150 \mathrm{~kg}=0,529 \mathrm{~kg}$ \\
\hline 0 derece atak açı & $\begin{array}{l}\text { Polistiren Köpük } \\
\text { Malzemesi }\end{array}$ & $\begin{array}{l}2 \text { Kat S Glass Kaplama /90 } \\
\text { derece fiber yönlü }\end{array}$ & $\begin{array}{l}\text { 2 Kat S Glass Kaplama /45 } \\
\text { derece fiber yönlü }\end{array}$ & $\begin{array}{l}2 \text { Kat S Glass Kaplama /0 } \\
\text { derece fiber yönlü }\end{array}$ \\
\hline Total Deformasyon & $72,39 \mathrm{~mm}$ & $1,83 \mathrm{~mm}$ & $3,12 \mathrm{~mm}$ & $4,64 \mathrm{~mm}$ \\
\hline \begin{tabular}{|l|l|} 
XYönündeki deformasyg \\
\end{tabular} & $0,36 \mathrm{~mm}$ & $0,054 \mathrm{~mm}$ & $0,083 \mathrm{~mm}$ & $0,074 \mathrm{~mm}$ \\
\hline \begin{tabular}{|l|l} 
Y Yönündeki deformasyg \\
\end{tabular} & $72,38 \mathrm{~mm}$ & $1,83 \mathrm{~mm}$ & $3,11 \mathrm{~mm}$ & $4,64 \mathrm{~mm}$ \\
\hline \begin{tabular}{|l|l|} 
ZYönündeki deformasyg \\
\end{tabular} & $2,73 \mathrm{~mm}$ & $0,022 \mathrm{~mm}$ & $0,093 \mathrm{~mm}$ & $0,165 \mathrm{~mm}$ \\
\hline Güvenlik Faktörü & 0,778 & 7,05 & 7,3 & 6,78 \\
\hline Kalınlık & & $0,30 \mathrm{~mm}$ & $0,30 \mathrm{~mm}$ & $0,30 \mathrm{~mm}$ \\
\hline Ağrrlık & $0,150 \mathrm{~kg}$ & $0,494 \mathrm{~kg}+0,150 \mathrm{~kg}=0,644 \mathrm{~kg}$ & $0,494 \mathrm{~kg}+0,150 \mathrm{~kg}=0,644 \mathrm{~kg}$ & $0,494 \mathrm{~kg}+0,150 \mathrm{~kg}=0,644 \mathrm{~kg}$ \\
\hline 0 derece atak açı & $\begin{array}{l}\text { Polistiren Köpük } \\
\text { Malzemesi }\end{array}$ & $\begin{array}{l}2 \text { Kat S Glass Kaplama /90 } \\
\text { derece fiber yönlü }\end{array}$ & $\begin{array}{l}2 \text { Kat S Glass Kaplama /45 } \\
\text { derece fiber yönlü }\end{array}$ & $\begin{array}{l}2 \text { Kat S Glass Kaplama /0 } \\
\text { derece fiber yönlü }\end{array}$ \\
\hline \begin{tabular}{|l} 
Total Deformasyon \\
\end{tabular} & $72,39 \mathrm{~mm}$ & $1,85 \mathrm{~mm}$ & $3,03 \mathrm{~mm}$ & $4,48 \mathrm{~mm}$ \\
\hline XYönündeki deformasyc & $0,36 \mathrm{~mm}$ & $0,056 \mathrm{~mm}$ & $0,077 \mathrm{~mm}$ & $0,074 \mathrm{~mm}$ \\
\hline Y Yönündeki deformasyg & $72,38 \mathrm{~mm}$ & $1,85 \mathrm{~mm}$ & $3,03 \mathrm{~mm}$ & $4,48 \mathrm{~mm}$ \\
\hline ZYönündeki deformasyd & $2,73 \mathrm{~mm}$ & $0,020 \mathrm{~mm}$ & $0,087 \mathrm{~mm}$ & $0,154 \mathrm{~mm}$ \\
\hline Güvenlik Faktörü & 0,778 & 6,24 & 6,66 & 6,31 \\
\hline
\end{tabular}

$100 \mathrm{G}$ için tek kat kaplama sonuçları aşağıda gösterilmiştir.

Tablo 7. 23 mm 1 kat Epoksi Karbon Kaplama

\begin{tabular}{|c|c|c|c|c|}
\hline Kalınlık & & $0,23 \mathrm{~mm}$ & $0,23 \mathrm{~mm}$ & $0,23 \mathrm{~mm}$ \\
\hline \multirow[t]{2}{*}{ Ağ̈rlık } & $0,150 \mathrm{~kg}$ & \multicolumn{3}{|c|}{$0,141 \mathrm{~kg}+0,150 \mathrm{~kg}=0,291 \mathrm{~kg} 0,141 \mathrm{~kg}+0,150 \mathrm{~kg}=0,291 \mathrm{~kg} 0,141 \mathrm{~kg}+0,150 \mathrm{~kg}=0,291 \mathrm{~kg}$} \\
\hline & $\begin{array}{l}\text { Polistiren Köpük } \\
\text { Malzemesi }\end{array}$ & $\begin{array}{l}\text { Kaplama/90 derece fiber } \\
\text { yönlü }\end{array}$ & $\begin{array}{l}\text { Kaplama / } 45 \text { derece fiber } \\
\text { yönlü }\end{array}$ & $\begin{array}{l}\text { Kaplama /0 derece fiber } \\
\text { yönlü }\end{array}$ \\
\hline Total Deformasyon & $147,73 \mathrm{~mm}$ & $2,452 \mathrm{~mm}$ & $5,500 \mathrm{~mm}$ & $8,779 \mathrm{~mm}$ \\
\hline XYönündeki deformasyo & $0,74 \mathrm{~mm}$ & $0,080 \mathrm{~mm}$ & $0,179 \mathrm{~mm}$ & $0,138 \mathrm{~mm}$ \\
\hline YYönündeki deformasyo & $0147,71 \mathrm{~mm}$ & $2,451 \mathrm{~mm}$ & $5,496 \mathrm{~mm}$ & $8,778 \mathrm{~mm}$ \\
\hline Z Yönündeki deformasyo & $5,58 \mathrm{~mm}$ & $0,020 \mathrm{~mm}$ & $0,191 \mathrm{~mm}$ & $0,330 \mathrm{~mm}$ \\
\hline Güvenlik Faktörü & 0,381 & 5,36 & 4,81 & 4,66 \\
\hline Kalınlık & & $0,30 \mathrm{~mm}$ & $0,30 \mathrm{~mm}$ & $0,30 \mathrm{~mm}$ \\
\hline \multirow[t]{2}{*}{ Ağırlık } & $0,150 \mathrm{~kg}$ & \multicolumn{3}{|c|}{$0,184 \mathrm{~kg}+0,150 \mathrm{~kg}=0,334 \mathrm{~kg} 0,184 \mathrm{~kg}+0,150 \mathrm{~kg}=0,334 \mathrm{~kg} 0,184 \mathrm{~kg}+0,150 \mathrm{~kg}=0,334 \mathrm{~kg}$} \\
\hline & $\begin{array}{l}\text { Polistiren Köpük } \\
\text { Malzemesi }\end{array}$ & $\begin{array}{l}\text { Kaplama/90 derece fiber } \\
\text { yönlü }\end{array}$ & $\begin{array}{l}\text { Kaplama/45 derece fiber } \\
\text { yönlü }\end{array}$ & $\begin{array}{l}\text { Kaplama /0 derece fiber } \\
\text { yönlü }\end{array}$ \\
\hline Total Deformasyon & $147,73 \mathrm{~mm}$ & $2,436 \mathrm{~mm}$ & $5,079 \mathrm{~mm}$ & $8,049 \mathrm{~mm}$ \\
\hline XYönündeki deformasyo & $0,74 \mathrm{~mm}$ & $0,081 \mathrm{~mm}$ & $0,158 \mathrm{~mm}$ & $0,130 \mathrm{~mm}$ \\
\hline YYönündeki deformasyo & $0147,71 \mathrm{~mm}$ & $2,435 \mathrm{~mm}$ & $5,075 \mathrm{~mm}$ & $8,048 \mathrm{~mm}$ \\
\hline ZYönündeki deformasyo & $5,58 \mathrm{~mm}$ & $0,017 \mathrm{~mm}$ & $0,170 \mathrm{~mm}$ & $0,292 \mathrm{~mm}$ \\
\hline Güvenlik Faktörü & 0,381 & 4,96 & 4,91 & 4,52 \\
\hline
\end{tabular}

Tablo 8. $23 \mathrm{~mm} 1$ kat Epoksi E Glass UD Kaplama

\begin{tabular}{|c|c|c|c|c|}
\hline Kalınlık & & $0,23 \mathrm{~mm}$ & $0,23 \mathrm{~mm}$ & $0,23 \mathrm{~mm}$ \\
\hline \multirow[t]{2}{*}{ Ä̆̈rlık } & $0,150 \mathrm{~kg}$ & $0,189 \mathrm{~kg}+0,150 \mathrm{~kg}=0,339 \mathrm{~kg}$ & $\mathrm{~g} 0,189 \mathrm{~kg}+0,150 \mathrm{~kg}=0,339 \mathrm{~kg}$ & $0,189 \mathrm{~kg}+0,150 \mathrm{~kg}=0,339 \mathrm{~kg}$ \\
\hline & $\begin{array}{l}\text { Polistiren Köpük } \\
\text { Malzemesi }\end{array}$ & $\begin{array}{l}1 \text { Kat Epoxy E Glass UD } \\
\text { Kaplama /90 derece fiber } \\
\text { yönlü }\end{array}$ & $\begin{array}{l}1 \text { Kat Epoxy E Glass UD } \\
\text { Kaplama / } / 45 \text { derece fiber } \\
\text { yönlü }\end{array}$ & $\begin{array}{l}1 \text { Kat Epoxy E Glass UD } \\
\text { Kaplama /0 derece fiber } \\
\text { yönlü }\end{array}$ \\
\hline Total Deformasyon & $147,73 \mathrm{~mm}$ & $3,945 \mathrm{~mm}$ & $6,567 \mathrm{~mm}$ & $9,193 \mathrm{~mm}$ \\
\hline XYönündeki deformasyo & $0,74 \mathrm{~mm}$ & $0,106 \mathrm{~mm}$ & $0,187 \mathrm{~mm}$ & $0,152 \mathrm{~mm}$ \\
\hline YYönündeki deformasyo & $147,71 \mathrm{~mm}$ & $3,944 \mathrm{~mm}$ & $6,564 \mathrm{~mm}$ & $9,192 \mathrm{~mm}$ \\
\hline ZYönündeki deformasyo & $5,58 \mathrm{~mm}$ & $0,069 \mathrm{~mm}$ & $0,222 \mathrm{~mm}$ & $0,334 \mathrm{~mm}$ \\
\hline Güvenlik Faktörü & 0,381 & 4,5 & 4,1 & 4 \\
\hline Kalınlık & & $0,30 \mathrm{~mm}$ & $0,30 \mathrm{~mm}$ & $0,30 \mathrm{~mm}$ \\
\hline \multirow[t]{2}{*}{ Ağırlık } & $0,150 \mathrm{~kg}$ & $0,247 \mathrm{~kg}+0,150 \mathrm{~kg}=0,397 \mathrm{~kg}$ & $\mathrm{~g} 0,247 \mathrm{~kg}+0,150 \mathrm{~kg}=0,397 \mathrm{~kg}$ & $0,247 \mathrm{~kg}+0,150 \mathrm{~kg}=0,397 \mathrm{~kg}$ \\
\hline & $\begin{array}{l}\text { Polistiren Köpük } \\
\text { Malzemesi }\end{array}$ & $\begin{array}{l}1 \text { Kat Epoxy E Glass UD } \\
\text { Kaplama /90 derece fiber } \\
\text { yönlü }\end{array}$ & $\begin{array}{l}1 \text { Kat Epoxy E Glass UD } \\
\text { Kaplama / } 45 \text { derece fiber } \\
\text { yönlü }\end{array}$ & $\begin{array}{l}1 \text { Kat Epoxy E Glass UD } \\
\text { Kaplama /0 derece fiber } \\
\text { yönlü }\end{array}$ \\
\hline Total Deformasyon & $147,73 \mathrm{~mm}$ & $3,848 \mathrm{~mm}$ & $6,177 \mathrm{~mm}$ & $8,608 \mathrm{~mm}$ \\
\hline XYönündeki deformasyo & $0,74 \mathrm{~mm}$ & $0,106 \mathrm{~mm}$ & $0,167 \mathrm{~mm}$ & $0,146 \mathrm{~mm}$ \\
\hline Y Yönündeki deformasyo & $147,71 \mathrm{~mm}$ & $3,847 \mathrm{~mm}$ & $6,174 \mathrm{~mm}$ & $8,607 \mathrm{~mm}$ \\
\hline Z Yönündeki deformasyo & $5,58 \mathrm{~mm}$ & $0,060 \mathrm{~mm}$ & $0,201 \mathrm{~mm}$ & $0,301 \mathrm{~mm}$ \\
\hline Güvenlik Faktörü & 0,381 & 4,21 & 3,97 & 3,79 \\
\hline
\end{tabular}

Tablo 9. $23 \mathrm{~mm} 1$ kat Epoksi S Glass UD Kaplama

\begin{tabular}{|c|c|c|c|c|}
\hline Kalınlık & & $0,23 \mathrm{~mm}$ & $0,23 \mathrm{~mm}$ & $0,23 \mathrm{~mm}$ \\
\hline \multirow[t]{2}{*}{ Ağrllık } & $0,150 \mathrm{~kg}$ & \multicolumn{3}{|c|}{$0,189 \mathrm{~kg}+0,150 \mathrm{~kg}=0,337 \mathrm{~kg} 0,189 \mathrm{~kg}+0,150 \mathrm{~kg}=0,339 \mathrm{~kg} 0,189 \mathrm{~kg}+0,150 \mathrm{~kg}=0,339 \mathrm{~kg}$} \\
\hline & $\begin{array}{l}\text { Polistiren Köpük } \\
\text { Malzemesi }\end{array}$ & $\begin{array}{l}1 \text { Kat S Glass Kaplama /90 } \\
\text { derece fiber yönlü }\end{array}$ & $\begin{array}{l}1 \text { Kat S Glass Kaplama /45 } \\
\text { derece fiber yönlü }\end{array}$ & $\begin{array}{l}1 \text { Kat S Glass Kaplama /0 } \\
\text { derece fiber yönlü }\end{array}$ \\
\hline Total Deformasyon & $147,73 \mathrm{~mm}$ & $3,778 \mathrm{~mm}$ & $7,119 \mathrm{~mm}$ & $10,799 \mathrm{~mm}$ \\
\hline XYönündeki deformasyo & $0,74 \mathrm{~mm}$ & $0,104 \mathrm{~mm}$ & $0,223 \mathrm{~mm}$ & $0,164 \mathrm{~mm}$ \\
\hline YYönündeki deformasyo & $147,71 \mathrm{~mm}$ & $3,777 \mathrm{~mm}$ & $7,114 \mathrm{~mm}$ & $10,798 \mathrm{~mm}$ \\
\hline 2 Yönündeki deformasyo & $5,58 \mathrm{~mm}$ & $0,061 \mathrm{~mm}$ & $0,235 \mathrm{~mm}$ & $0,417 \mathrm{~mm}$ \\
\hline Güvenlik Faktörü & 0,381 & 4,51 & 4,15 & 3,804 \\
\hline Kalınlık & & $0,30 \mathrm{~mm}$ & $0,30 \mathrm{~mm}$ & $0,30 \mathrm{~mm}$ \\
\hline \multirow[t]{2}{*}{ Ağrllık } & $0,150 \mathrm{~kg}$ & \multicolumn{3}{|c|}{$0,247 \mathrm{~kg}+0,150 \mathrm{~kg}=0,397 \mathrm{~kg} 0,247 \mathrm{~kg}+0,150 \mathrm{~kg}=0,397 \mathrm{~kg} 0,247 \mathrm{~kg}+0,150 \mathrm{~kg}=0,397 \mathrm{k}$} \\
\hline & $\begin{array}{l}\text { Polistiren Köpük } \\
\text { Malzemesi }\end{array}$ & $\begin{array}{l}1 \text { Kat S Glass Kaplama /90 } \\
\text { derece fiber yönlü }\end{array}$ & $\begin{array}{l}1 \text { Kat S Glass Kaplama /45 } \\
\text { derece fiber yönlü }\end{array}$ & $\begin{array}{l}1 \text { Kat S Glass Kaplama /0 } \\
\text { derece fiber yönlü }\end{array}$ \\
\hline Total Deformasyon & $147,73 \mathrm{~mm}$ & $3,692 \mathrm{~mm}$ & $6,687 \mathrm{~mm}$ & $10,07 \mathrm{~mm}$ \\
\hline \begin{tabular}{|l|l} 
YYönündeki deformasyo \\
\end{tabular} & $0,74 \mathrm{~mm}$ & $0,104 \mathrm{~mm}$ & $0,196 \mathrm{~mm}$ & $0,156 \mathrm{~mm}$ \\
\hline YYönündeki deformasyo & $147,71 \mathrm{~mm}$ & $3,691 \mathrm{~mm}$ & $6,683 \mathrm{~mm}$ & $10,069 \mathrm{~mm}$ \\
\hline ZYönündeki deformasyo & $5,58 \mathrm{~mm}$ & $0,054 \mathrm{~mm}$ & $0,213 \mathrm{~mm}$ & $0,378 \mathrm{~mm}$ \\
\hline Güvenlik Faktörü & 0,381 & 4,2 & 3,95 & 3,65 \\
\hline
\end{tabular}


İki kat kaplama sonuçları aşağıda gösterilmiştir.

Tablo 10. 23 mm 2 kat Epoksi Karbon Kaplama

\begin{tabular}{|c|c|c|c|c|}
\hline Kalınlık & & $0,23 \mathrm{~mm}$ & $0,23 \mathrm{~mm}$ & $0,23 \mathrm{~mm}$ \\
\hline Ağ̈rlık & $0,150 \mathrm{~kg}$ & $0,282 \mathrm{~kg}+0,150 \mathrm{~kg}=0,432 \mathrm{k}$ & $\mathrm{g} 0,282 \mathrm{~kg}+0,150 \mathrm{~kg}=0,432 \mathrm{k}$ & $0,282 \mathrm{~kg}+0,150 \mathrm{~kg}=0,432 \mathrm{~kg}$ \\
\hline 0 derece atak açıs & $\begin{array}{l}\text { Polistiren Köpük } \\
\text { Malzemesi }\end{array}$ & $\begin{array}{l}2 \text { Kat Epoksi Karbon } \\
\text { Kaplama /90 derece fiber } \\
\text { yönlü }\end{array}$ & $\begin{array}{l}2 \text { Kat Epoksi Karbon } \\
\text { Kaplama /45 derece fiber } \\
\text { yönlü }\end{array}$ & $\begin{array}{l}2 \text { Kat Epoksi Karbon } \\
\text { Kaplama/0 derece fiber } \\
\text { yönlü }\end{array}$ \\
\hline Total Deformasyon & $147,73 \mathrm{~mm}$ & $2,47 \mathrm{~mm}$ & $4,62 \mathrm{~mm}$ & $7,25 \mathrm{~mm}$ \\
\hline \begin{tabular}{|l|} 
XYönündeki deformasyo \\
\end{tabular} & $0,74 \mathrm{~mm}$ & $0,085 \mathrm{~mm}$ & $0,135 \mathrm{~mm}$ & $0,121 \mathrm{~mm}$ \\
\hline Y Yönündeki deformasyo & $0147,71 \mathrm{~mm}$ & $2,47 \mathrm{~mm}$ & $4,62 \mathrm{~mm}$ & $7,25 \mathrm{~mm}$ \\
\hline Z Yönündeki deformasyo & $5,58 \mathrm{~mm}$ & $0,013 \mathrm{~mm}$ & $0,147 \mathrm{~mm}$ & $0,247 \mathrm{~mm}$ \\
\hline Güvenlik Faktörü & 0,381 & 4,2 & 4,63 & 4,12 \\
\hline Kalınlık & & $0,30 \mathrm{~mm}$ & $0,30 \mathrm{~mm}$ & $0,30 \mathrm{~mm}$ \\
\hline Ä̈rrlık & $0,150 \mathrm{~kg}$ & $0,368 \mathrm{~kg}+0,150 \mathrm{~kg}=0,518 \mathrm{k}$ & $0,368 \mathrm{~kg}+0,150 \mathrm{~kg}=0,518 \mathrm{k}$ & $0,368 \mathrm{~kg}+0,150 \mathrm{~kg}=0,518 \mathrm{~kg}$ \\
\hline 0 derece atak açıs & $\begin{array}{l}\text { Polistiren Köpük } \\
\text { Malzemesi }\end{array}$ & $\begin{array}{l}2 \text { Kat Epoksi Karbon } \\
\text { Kaplama/90 derece fiber } \\
\text { yönlü }\end{array}$ & \begin{tabular}{|l}
2 Kat Epoksi Karbon \\
Kaplama /45 derece fiber \\
yönlü
\end{tabular} & $\begin{array}{l}2 \text { Kat Epoksi Karbon } \\
\text { Kaplama /0 derece fiber } \\
\text { yönlü }\end{array}$ \\
\hline Total Deformasyon & $147,73 \mathrm{~mm}$ & $2,52 \mathrm{~mm}$ & $4,43 \mathrm{~mm}$ & $6,92 \mathrm{~mm}$ \\
\hline XYönündeki deformasyo & $0,74 \mathrm{~mm}$ & $0,088 \mathrm{~mm}$ & $1,126 \mathrm{~mm}$ & $0,118 \mathrm{~mm}$ \\
\hline \begin{tabular}{|l|l} 
Y Yönündeki deformasyo \\
\end{tabular} & $147,71 \mathrm{~mm}$ & $2,52 \mathrm{~mm}$ & $4,43 \mathrm{~mm}$ & $6,92 \mathrm{~mm}$ \\
\hline 2 Yönündeki deformasyo & $5,58 \mathrm{~mm}$ & $0,012 \mathrm{~mm}$ & $0,137 \mathrm{~mm}$ & $0,226 \mathrm{~mm}$ \\
\hline Güvenlik Faktörü & 0,381 & 3,81 & 4,36 & 3,86 \\
\hline
\end{tabular}

Tablo 11. 23 mm 2 kat Epoksi E Glass Kaplama

\begin{tabular}{|c|c|c|c|c|}
\hline Kalınlık & & $0,23 \mathrm{~mm}$ & $0,23 \mathrm{~mm}$ & $0,23 \mathrm{~mm}$ \\
\hline Ağırlık & $0,150 \mathrm{~kg}$ & $0,379 \mathrm{~kg}+0,150 \mathrm{~kg}=0,529 \mathrm{k}$ & $0,379 \mathrm{~kg}+0,150 \mathrm{~kg}=0,529 \mathrm{k}$ & $\mathrm{kg} 0,379 \mathrm{~kg}+0,150 \mathrm{~kg}=0,529 \mathrm{~kg}$ \\
\hline 0 derece atak açıs & $\begin{array}{l}\text { Polistiren Köpük } \\
\text { Malzemesi }\end{array}$ & $\begin{array}{l}2 \text { Kat Epoxy E Glass UD } \\
\text { Kaplama/90 derece fiber } \\
\text { yönlü }\end{array}$ & $\begin{array}{l}\text { 2 Kat Epoxy E Glass UD } \\
\text { Kaplama /45 derece fiber } \\
\text { yönlü }\end{array}$ & $\begin{array}{l}2 \text { Kat Epoxy E Glass UD } \\
\text { Kaplama /0 derece fiber } \\
\text { yönlü }\end{array}$ \\
\hline Total Deformasyon & $147,73 \mathrm{~mm}$ & $3,81 \mathrm{~mm}$ & $5,77 \mathrm{~mm}$ & $8,00 \mathrm{~mm}$ \\
\hline \begin{tabular}{|l|} 
XYönündeki deformasyo \\
\end{tabular} & $0,74 \mathrm{~mm}$ & $0,109 \mathrm{~mm}$ & $0,145 \mathrm{~mm}$ & $0,140 \mathrm{~mm}$ \\
\hline Y Yönündeki deformasyo & $147,71 \mathrm{~mm}$ & $3,81 \mathrm{~mm}$ & $5,77 \mathrm{~mm}$ & $8,00 \mathrm{~mm}$ \\
\hline 2 Yönündeki deformasyo & $5,58 \mathrm{~mm}$ & $0,050 \mathrm{~mm}$ & $0,177 \mathrm{~mm}$ & $0,262 \mathrm{~mm}$ \\
\hline Güvenlik Faktörü & 0,381 & 3,52 & 3,67 & 3,47 \\
\hline Kalınlik & & $0,30 \mathrm{~mm}$ & $0,30 \mathrm{~mm}$ & $0,30 \mathrm{~mm}$ \\
\hline Ağrrlık & $0,150 \mathrm{~kg}$ & $0,494 \mathrm{~kg}+0,150 \mathrm{~kg}=0,644 \mathrm{k}$ & $0,494 \mathrm{~kg}+0,150 \mathrm{~kg}=0,644 \mathrm{k}$ & $0,494 \mathrm{~kg}+0,150 \mathrm{~kg}=0,644 \mathrm{k}$ \\
\hline 0 derece atak açıs & $\begin{array}{l}\text { Polistiren Köpük } \\
\text { Malzemesi }\end{array}$ & $\begin{array}{l}2 \text { Kat Epoxy E Glass UD } \\
\text { Kaplama/90 derece fiber } \\
\text { yönlü }\end{array}$ & \begin{tabular}{|l}
$\begin{array}{l}2 \text { Kat Epoxy E Glass UD } \\
\text { Kaplama/45 derece fiber } \\
\text { yönlü }\end{array}$ \\
\end{tabular} & $\begin{array}{l}\text { 2 Kat Epoxy E Glass UD } \\
\text { Kaplama /0 derece fiber } \\
\text { yönlü } \\
\end{array}$ \\
\hline Total Deformasyon & $147,73 \mathrm{~mm}$ & $3,848 \mathrm{~mm}$ & $6,177 \mathrm{~mm}$ & $8,608 \mathrm{~mm}$ \\
\hline \begin{tabular}{|l|} 
XYönündeki deformasyo \\
\end{tabular} & $0,74 \mathrm{~mm}$ & $0,106 \mathrm{~mm}$ & $0,167 \mathrm{~mm}$ & $0,146 \mathrm{~mm}$ \\
\hline YYönündeki deformasyo & $0147,71 \mathrm{~mm}$ & $3,847 \mathrm{~mm}$ & $6,174 \mathrm{~mm}$ & $8,607 \mathrm{~mm}$ \\
\hline ZYönündeki deformasyo & $5,58 \mathrm{~mm}$ & $0,060 \mathrm{~mm}$ & $0,201 \mathrm{~mm}$ & $0,301 \mathrm{~mm}$ \\
\hline Güvenlik Faktörü & 0,381 & 4,21 & 3,97 & 3,79 \\
\hline
\end{tabular}

Tablo 12. 23 mm 2 kat Epoksi S Glass Kaplama

\begin{tabular}{|c|c|c|c|c|}
\hline Kalınlık & & $0,23 \mathrm{~mm}$ & $0,23 \mathrm{~mm}$ & $0,23 \mathrm{~mm}$ \\
\hline Ag̈rllik & $0,150 \mathrm{~kg}$ & \multicolumn{3}{|c|}{$0,379 \mathrm{~kg}+0,150 \mathrm{~kg}=0,529 \mathrm{~kg} 0,379 \mathrm{~kg}+0,150 \mathrm{~kg}=0,529 \mathrm{~kg} 0,379 \mathrm{~kg}+0,150 \mathrm{~kg}=0,529 \mathrm{~kg}$} \\
\hline 0 derece atak açıs & $\begin{array}{l}\text { Polistiren Köpük } \\
\text { Malzemesi }\end{array}$ & $\begin{array}{l}2 \text { Kat S Glass Kaplama /90 } \\
\text { derece fiber yönlü }\end{array}$ & $\begin{array}{l}2 \text { Kat S Glass Kaplama/45 } \\
\text { derece fiber yönlü }\end{array}$ & $\begin{array}{l}2 \text { Kat } S \text { Glass Kaplama /0 } \\
\text { derece fiber yönlü }\end{array}$ \\
\hline Total Deformasyon & $147,73 \mathrm{~mm}$ & $3,67 \mathrm{~mm}$ & $6,24 \mathrm{~mm}$ & $9,28 \mathrm{~mm}$ \\
\hline \begin{tabular}{|l|} 
YYönündeki deformasyo \\
\end{tabular} & $0,74 \mathrm{~mm}$ & $0,108 \mathrm{~mm}$ & $0,167 \mathrm{~mm}$ & $0,149 \mathrm{~mm}$ \\
\hline YYönündeki deformasyo & $147,71 \mathrm{~mm}$ & $3,66 \mathrm{~mm}$ & $6,23 \mathrm{~mm}$ & $9,28 \mathrm{~mm}$ \\
\hline ZYönündeki deformasyo & $5,58 \mathrm{~mm}$ & $0,044 \mathrm{~mm}$ & $0,187 \mathrm{~mm}$ & $0,330 \mathrm{~mm}$ \\
\hline Güvenlik Faktörü & 0,381 & 3,52 & 3,65 & 3,39 \\
\hline Kalınlık & & $0,30 \mathrm{~mm}$ & $0,30 \mathrm{~mm}$ & $0,30 \mathrm{~mm}$ \\
\hline Ä̈rrlık & $0,150 \mathrm{~kg}$ & \multicolumn{3}{|c|}{$0,494 \mathrm{~kg}+0,150 \mathrm{~kg}=0,644 \mathrm{~kg} \mathrm{0} 0494 \mathrm{~kg}+0,150 \mathrm{~kg}=0,644 \mathrm{~kg} 0,494 \mathrm{~kg}+0,150 \mathrm{~kg}=0,644 \mathrm{~kg}$} \\
\hline 0 derece atak açı\$ & $\begin{array}{l}\text { Polistiren Köpük } \\
\text { Malzemesi }\end{array}$ & $\begin{array}{l}2 \text { Kat S Glass Kaplama /90 } \\
\text { derece fiber yönlü }\end{array}$ & $\begin{array}{l}2 \text { Kat S Glass Kaplama /45 } \\
\text { derece fiber yönlü }\end{array}$ & $\begin{array}{l}2 \text { Kat S Glass Kaplama /0 } \\
\text { derece fiber yönlü }\end{array}$ \\
\hline Total Deformasyon & $147,73 \mathrm{~mm}$ & $3,70 \mathrm{~mm}$ & $6,07 \mathrm{~mm}$ & $8,97 \mathrm{~mm}$ \\
\hline XYönündeki deformasyo & $0,74 \mathrm{~mm}$ & $0,112 \mathrm{~mm}$ & $0,154 \mathrm{~mm}$ & $0,148 \mathrm{~mm}$ \\
\hline YYönündeki deformasyo & $147,71 \mathrm{~mm}$ & $3,70 \mathrm{~mm}$ & $6,06 \mathrm{~mm}$ & $8,97 \mathrm{~mm}$ \\
\hline 2 Yönündeki deformasyo & $5,58 \mathrm{~mm}$ & $0,040 \mathrm{~mm}$ & $0,175 \mathrm{~mm}$ & $0,308 \mathrm{~mm}$ \\
\hline Güvenlik Faktörü & 0,381 & 3,12 & 3,33 & 3,15 \\
\hline
\end{tabular}

Yapılan analiz sonuçlarına göre model üzerine 50 G'lik ivme uygulandığında tek kat malzeme için bulgular;

- Kaplama malzemelerinin fiber açılarına göre deformasyonlara bakıldığında, üç kaplama malzemesi için de en yüksek deformasyonlar 0 derece fiber açısında yapılan analizlerde ortaya çıkmıştır.

- $50 \mathrm{G}$ ivme ile yapılan analizde göze çarpan noktalardan bir tanesi şu olmuştur; yukarıda belirtildiği gibi en fazla deformasyonun meydana geldiği malzeme tipi Epoxy S Glass UD olarak belirtilmişti lakin 90 derece fiber açısındaki deformasyonlarda Epoxy E Glass UD malzemesinin deformasyon değeri, Epoxy S Glass UD malzemesinde meydana gelen deformasyon değerinden daha fazla olduğu görülmüştür. Aynı zamanda güvenlik faktörüne bakıldığında Epoxy E Glass UD malzemesindeki değer, Epoxy S Glass malzemesindeki değerden daha yüksek olduğu görülmüştür. Burada beklenen sonuç, aynı kalınlıkta yapılan malzeme sonuçlarına göre deformasyon değeri yüksek olan malzeme tipinde güvenlik faktörünün daha düşük olmasıdır. Sonuçlar bize bunun tersini göstermiştir ve bu da kompozit malzemelerin yapısal olarak değişkenlik gösterdiğinin kanıtıdır.

Yapılan analiz sonuçlarına göre model üzerine 50 G'lik ivme uygulandığında iki kat malzeme için bulgular;

- $50 \mathrm{G}$ ivme uygulanarak tek kat malzeme kaplanarak yapılan analiz sonuçları ile karşılaştırıldığında her malzeme tipi için iki kat kaplama sonuçlarında deformasyon değerlerinin düştüğü görülmektedir.

- Her üç kaplama malzemesi için de ortak bir sonuç göze çarpmaktadır. 90 derece fiber açısı kullanılarak yapılan kaplama sonuçlarında; $0,23 \mathrm{~mm}$ ile yapılan kaplamalardaki deformasyonun, $0,30 \mathrm{~mm}$ ile yapılan kaplamadaki deformasyondan daha düşük olduğu görülmüştür. Bu sonuç da bize kompozit malzeme özelliklerinin değişken olduğunu göstermiştir.

Yapılan analiz sonuçlarına göre model üzerine 100 G'lik ivme uygulandığında tek kat malzeme için bulgular;

- $50 \mathrm{G}$ olarak uygulanan ivme değerine göre $100 \mathrm{G}$ ivme değerinde deformasyon değerlerinde artış meydana gelmiştir ve buna bağlı olarak güvenlik faktöründe azalmalar görülmüştür.

- Kaplanan malzeme kalınlığı arttıkça deformasyon değerlerinde düşüş meydana gelmiş ve malzeme kütlesindeki artış nedeniyle de güvenlik faktörlerinde azalma görülmüştür.

Yapılan analiz sonuçlarına göre model üzerine 100 G'lik ivme uygulandığında iki kat malzeme için bulgular;

- $100 \mathrm{G}$ ivme uygulanarak tek kat malzeme kaplanarak yapılan analiz sonuçları ile karşılaştırıldığında her malzeme tipi için iki kat kaplama sonuçlarında deformasyon değerlerinin düştüğü görülmektedir.

- Epoxy Carbon UD malzemesinde 90 derece fiber açısında yapılan analizde $0,30 \mathrm{~mm}$ kalınlıkta, $0,23 \mathrm{~mm}$ kalınlığa göre deformasyonun daha yüksek olduğu görülmüştür. Kompozit malzemelerin farklı özellik göstermesinin neticesinde bu sonuca ulaşılmıştır.

- Epoxy E Glass UD malzemesinde; 0 derece, 45 derece ve 90 derece fiber açılarında yapılan analizlerde ise tüm fiber açılarında 0,30 mm kalınlıktaki deformasyon değerlerinin, $0,23 \mathrm{~mm}$ kalınlıktaki deformasyon değerlerinden daha yüksek olduğu görülmüştür. Aynı şekilde kompozit malzemenin farklı davranışlar sergilemesinin bir sonucu olarak bu veriler elde edilmiştir.

Epoxy S Glass UD malzemede ise Epoxy Carbon UD malzemesiyle benzer olarak sadece 90 derece fiber açısında 0,30 $\mathrm{mm}$ kaplamadaki deformasyonun $0,23 \mathrm{~mm}$ kaplamadaki deformasyondan daha yüksek olduğu görülmüştür. Kompozit malzmenin farklı özellik sergilemesi sonucunda aynı şekilde sonuca ulaşılmıştır.

\section{A. Kompozit Malzemelerin Avantajları}

- Yüksek mukavemet : Kompozit malzemeler yüksek mukavemete sahip malzemeler arasında en etkin olanlardan biridir. 
- Hafiflik : Kompozitler, takviyesiz plastikler ve metallere göre birim alan ağırlığında daha yüksek mukavemet değerlerine sahiptir.

- Tasarım esnekliği : Kompozitler her türlü basit, karmaşık, geniş, küçük, yapısal, estetik, dekoratif yada fonksiyonel amaçlı olarak tasarlanabilir.

- Boyutsal stabilite : Termoset kompozitler çeşitli mekanik baskılar altında şekillerini ve işlevselliklerini korurlar.

- Yüksek Dielektrik Direnimi : Kompozitler elektrik yalıtım özellikleri sayesinde göze çarpan malzemelerdir. birçok komponent'in üretiminde tercih sebebidir.

- Korozyon dayanımı : Kompozitler antikorozif özellikleri sayesinde diğer malzemelerden daha üstündür.

- Kalıplama kolaylığı : Kompozit ürünler, kalıplama işleminin tek parçada yapılması nedeniyle çelik gibi geleneksel malzemelerde karşılaşılan birçok parçanın birleştirilmesi ve sonradan monte edilmesi gibi işlemleri ortadan kaldırmaktadır.

- Yüzey uygulamaları : Kompozit ürünlerde kullanılan polyester reçine, özel pigment katkıları ile renklendirilerek, kendinden renkli olarak da üretilebilir.

- Şeffaflık özelliği : Kompozitler, cam kadar 1şık geçirgen olabilir. Tam şeffaf olması nedeni ile ışı̆̆ı yayması sayesinde, diffüze ışı̆̆ın önem kazandığı seralarda ve güneş kolektörü yapımında önemli avantaj sağlar.

- Beton yüzeylere uygulama imkanı : Kompozitler beton yüzeylere iyi yapışır. Özellikle, betonun gözenekli olması nedeniyle, kompoziti oluşturan ana malzemelerden polyester reçinenin beton gözeneklerinden sızması ve beton kütle içinde sertleşmesinden dolayı mükemmel bir yapışma meydana gelir.

Ahşap yüzeylere uygulama imkanı : Kompozitlerin ahşap yüzeylere yapışma özelliği vardır. Bunun için ahşap malzemenin kuru olması gerekir. Ayrıca stiren ihtiva eden polyester reçine ile iyi bir şekilde emdirilmesi gereklidir.

- Demir yüzeylere uygulama imkanı : Demir yüzeydeki pas ve yağ kalıntıları temizlendikten sonra kompozitlerle kaplanabilir ve böylece demir ve çelik yüzeyler, korozyon etkilerinden korunurlar.

- Yanmazlık özelliği : Kompozitlerin aleve karşı dayanımı, kullanılan polyesterin özelliğine bağlıdır. Alev dayanım özelliği isteniyorsa "Alev dayanımlı" polyester seçilmelidir.

- Kompozitler sicaklıktan etkilenmez : Kompozit ürünler, termoset plastikler grubundan polyester reçineler ile yapıldığ

\section{Sonuç}

Analiz sonuçları göstermektedir ki; malzeme özelliklerine bağlı olarak her bir parametre analiz sonuçlarına etki etmektedir. Kompozit malzeme kullanılarak yapılan analizlerde, malzeme üzerinde oluşan deformasyonların gözle görülür şekilde düşmesi bizlere konpozit malzemenin önemini bir kez daha göstermiştir.

Ayrıca kompozit malzemelerin faklı davranışlar göstermesinin neticesi olarak bu özellikler malzeme üzerinde kompozit olarak kullanılacak bölgelerin bölümlere ayrılarak, malzemenin gösterdiği karakteristiğe göre de ilgili bölgede kullanım olanağ sağlamaktadır.

Bir diğer çıkarılan sonuç ise, kompozit malzemelerin farklı karakteristik göstermesi neticesinde, kullanılması planlanan malzemenin, model üzerindeki yere bağlı olarak çok daha fazla araştırılması ve analiz yapılması gerekliliğidir. yumuşamaz ve şekil değiştirmez. Isı dayanıklılığı kullanılan polyester reçinenin cinsine bağlıdır.

- Kompozitler içine farklı malzemeler gömülebilir : Kompozitlerin içine demir, ahşap, halat, tel, mukavva, poliüretan sert köpük gibi malzemeler gömülerek mekanik özellikleri değiştirilebilir.

- Tamir edilebilirlik özelliği : Tamir işleri bir kalıp üzerinde yapılırsa tamir izleri görünmez veya tamir sonrası boya veya zımpara yapılması gerekebilir.

- Kompozitler kesilip delinebilir : Kompozitler, tahta gibi kolayca kesilir, delinir, zımparalanır. $\mathrm{Bu}$ amaçla kullanılan aletlerin sert çelik veya elmas uçlu olması halinde daha iyi sonuç alınmaktadır.

- Çatlak ilerlemesi olayı minimize edilmiştir.

- Titreşimleri absorbe edilme özelliği sağlanmıştır.

- Kompozitlerden bazıları çok yüksek akma sınırı (akma gerilmesi) değerlerine sahiptir. [40]

\section{B. Kompozit Malzemelerin Dezavantajları}

- Metallere yapışmazlar. Firınlamadan (pişirmeden) kullanılamazlar.

- Değişik doğrultularda değişik mekanik özelliklere sahiptir.

- Aynı kompozit malzemeler için çekme, basma, kesme, eğilme mukavemet değerleri farklıdır

- Elyaf doğrultusundaki elastik modülü, elyafa dik doğrultudaki elastik modülünden daha büyüktür.

- Üretimi pahalıdır.

- Nem ve hava zerrecikleri, kompozitlerin mekanik ve yorulma özelliklerini olumsuz yönde etkiler.

- Delik delme ve kesme türü işlemler liflerde açılmaya yol açmaktadır

- Malzeme maliyeti yüksektir

- Belirlenmiş dizayn kurallarının eksikliği

- Metal ve kompozit tasarımları nadiren değiştirilebilir

- Geliştirme süreleri uzundur

- Birleştirici, vida/civata v.b., var olduğu için maliyetlidir

- Solvent/nem etkisi

- Kullanılabildiği sıcaklık limitleri

- Darbelere karşı hassastırlar [41]

\section{Kaynakça}

[1]Kong, C., Bang, J., Sugiyama, Y., Structural Investigation of Composite Wind Turbine Blade Considering Various Load Cases and Fatigue Life, Energy, Vol.30, Pages 2101-2114, 2005

[2]Bechly, M.E., Clausen, P.D., Structural Design of a Composite Wind Turbine Blade Using Finite Element Analysis, Computers \& Structures, Vol. 63, Pages 639-646, 1997.

[3] Avcı, B., Yılmaz, T.B., Rüzgar Türbini Kanat Tasarımı ve Analizi, Bitirme Projesi, Dokuz Eylül Üniversitesi Mühendislik Fakültesi Makine Mühendisliği Bölümü, İzmir, 2012. sf.5-37

[4] Gülbahar, Ö.,"Karayel İnsansız Hava Aracının Statik Ve Dinamik Analizleri”, Yüksek Lisans Tezi, İstanbul Teknik Üniversitesi Fen Bilimleri Enstitüsü, İstanbul, 2-4 (2015).

[5] Vardar, A. ve Eker, B., "Rüzgar Türbinlerinde Uygun kanat Tipinin Seçilmesi".2004

[6] Scherer, R., Blade Design Aspects, Renewable Energy,Vol.16, Pages 1272-1277, 1999. 
[7]Habali, S.M., Saleh, I.A., Local Design, Testing and Manufacturing of Small Mixed Airfoil Wind Turbine Blades of Glass Fiber Reinforced Plastics Part I: Design of the Blade and Root, Energy Conversion \& Management, Vol. 41, Pages 249-280, 2000.

[8]Habali, S.M., Saleh, I.A., Local Design, Testing and Manufacturing of Small Mixed Airfoil Wind Turbine Blades of Glass Fiber Reinforced Plastics Part II: Manufacturing of Blade and Rotor, Energy Conversion \& Management, Vol.41, Sf. 281-298, 2000.

[9] Yang, J., Peng, C., Xiao, J., Zeng, J., Xing, S., Jin, J., Deng, H., Structural Investigation Of Composite Wind Turbine Blade Considering Structural Collapse İn Full-Scale Static Tests, Composite Structures, Vol.97, 15-29, 2013.

[10] Grogan, D.M., Leen, S.B., Kennedy, C.R., Bradaigh, C.M., Design of Composite Tidal Turbine Blades, Renewable Energy, Vol.57, 151-162, 2013.

[11] Tseng, Y.C., Kuo, C.Y., Engineering and Construction Torsional Responses of Glass-Fiber/Epoxy Composite Blade Shaft for a Small Wind Turbine, Procedia Engineering,Vol. 14, Pages 1996-2002, 2011.

[12] Uysal, A., Rüzgar Türbini Kanat Malzemelerinin Mekanik Özelliklerinin İncelenmesi, Yüksek Lisans Tezi, Yıldız Teknik Üniversitesi Fen Bilimleri Enstitüsü, İstanbul, 2008.

[13]Shokrieh, M.M., Rafiee, R., Simulation of Fatigue Failure in a Full Composite Wind Turbine Blade, Composite Structures, vol. 74, 332-342, 2006.

[14] Chen, X., Zhao, W., Zhao, X.L., Xu, J.Z., Preliminary Failure Investigation of a $52.3 \mathrm{~m}$ Glass/Epoxy Composite Wind Turbine Blade, Engineering Failure Analysis, 44, 345-350, 2014.

[15]https://dergipark.org.tr/tr/download/articlefile/400203"Selçuklu.ÜniversitesiMüh. Bilim ve Tekn. Derg., c.5, s.4, ss. 511-535, 2017"

[16]İnternet: Karaağaç, C., "İnsansız Hava Aracı Sistemleri: Gökyüzünün Yeni Yırtıcı Kuşları”, https://www.academia.edu/2004387/İnsans1z_Hava_Arac1_ Sistemleri_Gökyüzünün_Yeni_Yırtıc1_Kuşlār1 (2004).sf 1/8

[17]https://www.academia.edu/9199556/\%C4\%B0nsans\%C4\% B1z_Hava_Ara\%C3\%A7lar\%C4\%B1_Muharebe_Alan\%C4 $\%$ B1nda_ve_Ter\%C3\%B6rle_M\%C3\%BCcadelede_Devri msel_D\%C3\%B6n\%C3\%BC\%C5\%9F\%C3\%BCm_?auto=d ownload

[18]https://dergipark.org.tr/tr/download/article-file/1167969

[19] Orkan Özcan Deniz Bilge Demir Semih Sami Akay İha araçları ve Yer Bilimleri Kitab1 OCAK/2019 sf.10-20

[20] 14. ULUSLARARASI MEB ROBOT YARIŞMASI INSANSIZ HAVA ARACI (Mini Drone) KATEGORİII YARIŞMA KURALLARI, Şanlıurfa,2021

[21] Sivil Havacılık Genel Müdürlüğü-İnsansız Hava Aracı Talimat1(SHT-İHA) $\quad 2016 \quad 22 / 02 / 2016 \quad$ sf.4 http://web.shgm.gov.tr/documents/sivilhavacilik/files/mevzu at/sektorel/talimatlar/2019/IHA_talimati_revizyon3.pdf

[22] ElKholy, H., 2014. Dynamic Modeling and Control of a Quadrotor Using Linear and Nonlinear Approaches, Master Thesis, The American University In Cairo, School of Sciences and Engineering, Cairo, pp. (6-12, 64).

[23]Geleceğin Hava Kuvvetleri:2016-2050 Sektör Değerlendirme Raporui Şubat'16, Future Technology Institute

[24] İnsansız Hava Araçlarının Kullanım Alanları ve Gelecekteki Beklentiler Ekim/2012 https://www.researchgate.net/publication/292669083_Insans
iz_Hava_Araclarinin_Kullanim_Alanlari_ve_Gelecekteki_B eklentiler(Eisenbeiss, H. 2009). Sf.4

[25] ISSN: 1307-9905 E-ISSN: 2602-2133 Sayı Issue 32, Cilt Volume 12, 2021-1, 242-267 DOI: 10.31198/idealkent. 817201

[26] İnsansız Hava Araçları Ercan CANER-212 Kış Cilt: 1 Sayı: 1 Millî Güvenlik ve Askerî Bilimler Kış 2013-2014 • Cilt: 1 - Say1: 222-225

[27]SAKARYA MESLEK YÜKSEKOKULU MAKINA VE METAL TEKNOLOJILERİ BÖLÜMÜ METALURJI PROGRAMI, KOMPOZITT MALZEMLER DERS NOTU, ONAT A., Sakarya, 2015

[28]https://www.muhendisbeyinler.net/kompozit-malzemelerve-kullanim-alanlari/

[29] Yrd. Doç. Dr. Ali İhsan Kaya-Mehmet Akif Ersoy Üniversitesi Temmuz/2016

[30]https://arkipedia.net/kompozit-malzemelerin-ozellikleri-vesiniflandirilmasi/

[31]https://www.serdarkorkut.com/2017/01/22/kompozitmalzemeler/

[32]https://docplayer.biz.tr/68711544-Kompozit-malzemeler-vemekanigi-yrd-doc-dr-akin-atas.html

[33]https://tolgakaranfil.webnode.com.tr/products/kompozitmalzemeler\%2C-

s\%C4\%B1n\%C4\%B1fland\%C4\%B1r\%C4\%B11mas\%C4\% B1-ve-uretimi/

[34] http://www.plastik-ambalaj.com/tr/plastik-ambalajmakale/1019-termoplastik-ve-termoset-plastikler

[35] http://accluster.com/kompozit-malzemelerin-yapisi/

[36] https://www.bilgiustam.com/kompozit-malzemelerhakkinda-hersey/

[37]https://www.ceylankompozit.com/tr/\%C3\%BCretimdekullan\%C4\%B1lan-malzemeler 33

[38]http://teknikbil.blogspot.com/2013/11/polimer-malzemelerve-ozellikleri.html

[39] Nano Kompozitler

[40]https://www.solverkimya.com/site/makaleler/detaylar/komp ozit-malzeme.html

[41]https://www.metalurjik.com/kompozit-malzemelerinavantaj-ve-dezavantajlari

[42] HALİL İBRAHIM İŞLEK · PUBLİSHED AĞUSTOS 16, $2020 \cdot$ UPDATED AĞUSTOS 18, 2020

[43] https://kompozitkimya.com.tr/kompozit-nedir/

[44] STATİK YÜKLER ALTINDA BİR A320 UÇAK KANADININ KOMPOZIT MALZEMELER İLE TASARIMI VE ANALİİ, Günay Ö., Gaziantep Üniversitesi Yüksek Lisans Tezi, 2019

[45] https://www.ceyrekmuhendis.com/sonlu-elemanlar-analizinedir/

[46]https://kalkinmaguncesi.izka.org.tr/index.php/2021/08/04/ha vacilik-sektorunde-kompozit-malzeme-kullanimi/

[47]https://www.tusas.com/urunler/iha/operatif-stratejik-ihasistemleri/anka

[48] https://www.militaryfactory.com/

[49] https://www.aircraftnerds.com/2016/06/aircraft-wingconfiguration.html

[50] https://tr.esc.wiki/wiki/NACA_airfoil 